Sierra Leone is a developing country in W. Africa. Medical facilities are rudimentary. There was no gastroscopy service till 2016, when supported by a grant from the British Society of Gastroenterology (BSG), 4 gastroenterologists from northeast UK travelled to the capital, Freetown. We trained 3 doctors to perform gatroscopy (OGD). Since then we have supported the doctors remotely and by annual visits. They have done procedures in private hospital that owns endoscopy stack.

Aims No endoscopy equipment exists in Government sector. We felt that we should demonstrate the need for a service by concentrating on the management of a distinct clinical entity, AUGIB, in which the benefit of OGD is clear. We undertook an audit of UGIB presenting to the main government hospital in Freetown (Connaught Hospital) to look at clinical presentation and outcomes.

Method 2 house officers (HO's) allocated to collect data. The audit was publicised at weekly medical meetings and also, details were put in the hospital medical WhatsApp group. HO's visited outpatient department regularly to identify patients. Then, as record keeping is paper based and poor, they visited regularly to keep a record of clinical details results and outcomes. A proforma developed by the UK team and head of medicine was used to record demographic clinical and outcome details. Details from the proforma were transcribed on to an excel spreadsheet.

Results 24 patients identified in the period. 63.6\% male. Median age- 45 (26-67); Symptom duration at presentation 3 days (0.5-28). 10 patients had haematemesis, 2 melaena and 12 with both. None taking anticoagulant/antiplatetelet drugs. 2 patients on non NSAID. Significant comorbidities as follows; Hep B +ve - 2 (one clinically cirrhotic); HIV +ve - 1. One patient had previous AUGIB.

Clinical parameters on admission as follows: Median haemoglobin - $5 \mathrm{~g} / \mathrm{dl}$ (2-13.5); Median systolic BP - 98.5 mmHg (60-224); Median pulse - 115/min (80-146).

21 patients had at least one dose of IV Omeprazole. 15 patients had at least 1 unit of blood ((median $\mathrm{Hb}=5$ (27.3)). A single patient (Female aged 56) with admission $\mathrm{Hb}$ of $5 \mathrm{~g} / \mathrm{dL}$ underwent OGD day 7 of admission: Diagnosis - erosive oesophagitis.

15 patients discharged after mean 11.3 days (5-27). 8 patients died giving crude mortalty $-3.4 \%$. Mean time to death 3. Days (0-12).

Conclusion This 1st audit of outcomes of AUGIB in Sierra Leone shows a comparatively high mortality in relatively young patients. Reasons for this probably multifactorial. However, lack of access to endoscopy probably a contributory factor. We have developed a AUGIB bundle which we hope to administer whilst waiting for the government to invest in endoscopy equipment.

\section{PTU-064 ENDOSCOPIC RESECTION OF NON-AMPULLARY DUODENAL LESIONS: FEASIBILITY, SAFETY AND EFFICACY}

L Varytimiadis, E Hossain*, S Subramaniam, S Thayalasekaran, K Kandiah, A Alkandari, P Bhandari. Queen Alexandra Hospital, Portsmouth

10.1136/gutjnl-2019-BSGAbstracts.280
Introduction Non-ampullary duodenal adenomas (sporadic and duodenal lesions associated with familial adenomatous polyposis or FAP) are rare, with most lesions found incidentally on gastroscopy.

Endoscopic resection of duodenal lesions is increasingly being used in place of invasive surgery. However, endoscopic resection in the duodenum can be challenging due to its anatomy and may be associated with a higher risk of complications. This study aims to evaluate the safety and efficacy of endoscopic resection of duodenal polyps.

Methods All patients with large $(>10 \mathrm{~mm})$ non-ampullary duodenal polyps who underwent endoscopic resection between February 2008 and January 2019 in a single tertiary referral centre were included in the study. A retrospective analysis on data including demographics, size, histology, location, method of resection and complications was performed.

Results There were a total of 85 patients with polyps $>10$ $\mathrm{mm}$ referred for endoscopic therapy (median age 64 years, [IQR] 27-87 years, 52\% male). 58 (68\%) underwent endoscopic mucosal resection (EMR) and 27 (32\%) had knife assisted resection (KAR). Histology revealed 67 (79\%) LGD and $11(13 \%)$ HGD. Majority of the lesions (70) were found in D2 $(82 \%) .8$ patients $(9.4 \%)$ had a diagnosis of FAP.

The mean polyp size was $25.3 \mathrm{~mm}$ [IQR 10-80]. 80 polyps $(94.1 \%)$ were removed in a single session with an en-bloc resection rate of $40 \%$. The overall recurrence, bleeding and perforation rate was $7 \%, 8 \%$ and $2 \%$ respectively.

Table 1 shows the complication and recurrence rates stratified by lesion size $(<30 \mathrm{~mm}$ vs $>30 \mathrm{~mm})$.

\begin{tabular}{llll}
\multicolumn{2}{c}{ Abstract PTU-064 Table 1 } & & \\
\hline Lesion size & $<30 \mathrm{~mm}$ & $>30 \mathrm{~mm}$ & P value \\
\hline Number & 53 & 32 & \\
Bleeding & $2(4 \%)$ & $5(15 \%)$ & $\mathrm{p}=<0.001^{*}$ \\
Recurrence & $1(2 \%)$ & $5(16 \%)$ & $\mathrm{p}=<0.015^{*}$ \\
Perforation & $1(2 \%)$ & $1(3 \%)$ & $\mathrm{p}=0.62$ \\
\hline${ }^{*} \mathrm{p}<0.05$. & & &
\end{tabular}

All complications were managed endoscopically. The median follow up period was 38 months [IQR 4-120] and a higher rate of recurrence $(16 \%)$ was noted in lesions $>30 \mathrm{~mm}$. The majority (4/6) of the recurrences were treated successfully with subsequent endoscopic resection. The complication and recurrence rate was similar in patients undergoing EMR and KAR. No procedure related mortality was noted in this cohort.

Conclusions Endoscopic resection of duodenal lesions is a safe and effective technique for complete removal of these polyps. Lesion assessment is crucial in patient selection for endoscopic removal as noted by the absence of cancers in this cohort. The risk of complications, particularly bleeding is higher in lesions greater than $3 \mathrm{~cm}$. Similarly, recurrence rates are also higher in this group. Nevertheless, we demonstrated that all complications and most recurrences were successfully managed endoscopically. 